MS-TP-12-13

LPT-Orsay/12-101

CERN-PH-TH/2012-256

HU-EP-12/31

TCDMATH 12-08

DESY $12-167$

SFB/CPP-12-74

\title{
B-physics from non-perturbatively renormalized HQET in two-flavour lattice QCD
}

\author{
F $L P H A$ \\ Collaboration
}

Fabio Bernardoni ${ }^{\mathrm{a}}$, Benoît Blossier ${ }^{\mathrm{b}}$, John Bulava ${ }^{\mathrm{c}}$, Michele Della Morte ${ }^{\mathrm{d}}$, Patrick Fritzsch ${ }^{\mathrm{e}}$, Nicolas Garron $^{\mathrm{f}}$, Antoine Gérardin ${ }^{\mathrm{b}}$, Jochen Heitger ${ }^{\mathrm{g}, *}$, Georg M. von Hippel ${ }^{\mathrm{d}}$, Hubert Simma ${ }^{\mathrm{a}}$

${ }^{a}$ NIC, DESY, Platanenallee 6, D-15738 Zeuthen, Germany

${ }^{b}$ CNRS et Université Paris-Sud XI, Laboratoire de Physique Théorique, Bâtiment 210, F-91405 Orsay Cedex, France ${ }^{c}$ CERN, Physics Department, TH Unit, CH-1211 Geneva 23, Switzerland

dJohannes Gutenberg Universität Mainz, Institut für Kernphysik, Becher Weg 45, D-55099 Mainz, Germany

${ }^{e}$ Humboldt Universität Berlin, Institut für Physik, Newtonstraße 15, D-14289 Berlin, Germany

${ }^{f}$ Trinity College, School of Mathematics, Dublin 2, Ireland

${ }^{g}$ Westfälische Wilhelms-Universität Münster, Institut für Theoretische Physik, Wilhelm-Klemm-Straße 9, D-48149 Münster, Germany

\begin{abstract}
We report on the ALPHA Collaboration's lattice B-physics programme based on $N_{\mathrm{f}}=2 \mathrm{O}(a)$ improved Wilson fermions and HQET, including all NLO effects in the inverse heavy quark mass, as well as non-perturbative renormalization and matching, to fix the parameters of the effective theory. Our simulations in large physical volume cover 3 lattice spacings $a \approx(0.08-0.05) \mathrm{fm}$ and pion masses down to $190 \mathrm{MeV}$ to control continuum and chiral extrapolations. We present the status of results for the b-quark mass and the $\mathrm{B}_{(\mathrm{s})}$-meson decay constants, $f_{\mathrm{B}}$ and $f_{\mathrm{B}_{\mathrm{s}}}$.
\end{abstract}

\section{B-physics and lattice QCD}

Lattice simulations of QCD have established as a sound tool to compute strong interaction effects for accurate phenomenology in heavy flavour physics. For B-meson weak decays, which constrain the CKM Unitarity Triangle, lattice QCD results for the involved low-energy hadronic matrix elements in conjunction with experimental studies decisively contribute to stringent tests of the self-consistency of the Standard Model and complement direct searches for New Physics. Since the significance of such precision tests in the beauty sector is predominantly limited by theoretical uncertainties, lattice computations with an overall accuracy of a few \% are highly desirable. Let us highlight the " $V_{\mathrm{ub}}-$ puzzle" illustrated in Fig. 1. which has drawn the community's attention in the recent past. $\left|V_{\mathrm{ub}}\right|$ can be determined from inclusive semi-leptonic processes $\mathrm{B} \rightarrow X_{\mathrm{u}} \ell v$, from exclusive semi-leptonic $\mathrm{B} \rightarrow \pi \ell v$ decays and from the leptonic one, $\mathrm{B} \rightarrow \tau v$. In the latter two, the hadronic form

\footnotetext{
*Speaker at QCD 2012, Montpellier, France, 2 - 6 July 2012

Email address: heitger@uni-muenster.de (Jochen Heitger)
}

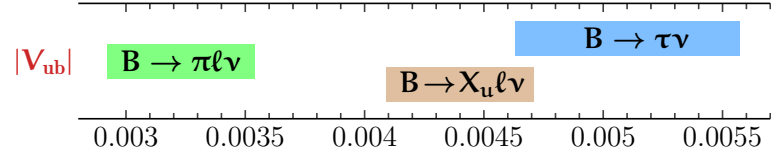

Figure 1: Observed tension among different $\left|V_{\mathrm{ub}}\right|$-determinations [1]; $\pm 1 \sigma$ bands are shown.

factor $f_{+}\left(q^{2}\right)$ and the B-meson decay constant $f_{\mathrm{B}}$ enter, respectively, so that lattice $\mathrm{QCD}$ input is required to extract $\left|V_{\mathrm{ub}}\right|$. As currently there is a $\sim 3 \sigma$ tension between its two exclusive (semi-leptonic and leptonic) determinations 1 as well as an inconsistency with the estimate from inclusive decays, precision lattice QCD calculations can contribute to resolve this tension [4-6].

\footnotetext{
${ }^{1}$ At the ICHEP 2012 Conference, the Belle Collaboration reported a new result for $\mathscr{B}(\mathrm{B} \rightarrow \tau v)[2] 3$, obtained on basis of a new data set using a more sophisticated tagging of the B. Taken alone, this would yield a value for $\left|V_{\mathrm{ub}}\right|$ compatible with the exclusive semi-leptonic determination. However, this result has not yet been confirmed by other collaborations, and more data and a careful study of all systematics are still required before drawing final conclusions.
} 


\section{Non-perturbative HQET}

The particular challenge of B-physics on the lattice lies in the many disparate scales, ranging from its inverse extent (as IR cutoff) over the hierarchy of differently flavoured hadron masses up to the inverse of the lattice spacing $a$ (as UV cutoff), to be treated simultaneously in the numerical simulations. Since lattice sizes that are computationally manageable today have $a m_{\mathrm{b}}>1$, discretization effects get most severe for heavy quark systems with b-quarks and escape brute force simulations. Our approach to lattice B-physics is therefore based on the Heavy Quark Effective Theory (HQET) for the bquark [7, 8], which consists in a systematic expansion of its QCD action and heavy-light correlation functions in $\Lambda_{\mathrm{QCD}} / m_{\mathrm{b}} \ll 1$ around the static limit $\left(m_{\mathrm{b}} \rightarrow \infty\right)$.

The Lagrangian entering the heavy quark field's lattice action $S_{\mathrm{HQET}}=a^{4} \sum_{x} \mathscr{L}_{\mathrm{HQET}}(x)$ in HQET at NLO, i.e., including $\mathrm{O}\left(1 / m_{\mathrm{b}}\right)$ terms, reads:

$$
\mathscr{L}_{\mathrm{HQET}}=\bar{\psi}_{\mathrm{h}} D_{0} \psi_{\mathrm{h}}-\omega_{\mathrm{kin}} O_{\mathrm{kin}}-\omega_{\text {spin }} O_{\text {spin }},
$$

with $\psi_{\mathrm{h}}$ satisfying $P_{+} \psi_{\mathrm{h}}=\psi_{\mathrm{h}}, P_{+}=\frac{1+\gamma_{0}}{2}$. The parameters $\omega_{\text {kin }}$ and $\omega_{\text {spin }}$ are formally $\mathrm{O}\left(1 / m_{\mathrm{b}}\right)$ and multiply the dimension-5 operators $O_{\text {kin }}=\bar{\psi}_{\mathrm{h}} \mathbf{D}^{2} \psi_{\mathrm{h}}$ and $O_{\text {spin }}=\bar{\psi}_{\mathrm{h}} \sigma \mathbf{B} \psi_{\mathrm{h}}$, representing interaction terms due to the motion and the spin of the heavy quark. Thus, $S_{\text {HQET }}$ has $\mathrm{O}\left(\Lambda_{\mathrm{QCD}}^{2} / m_{\mathrm{b}}^{2}\right)$ truncation errors, and lattice artifacts only scale as $\left(a \Lambda_{\mathrm{QCD}}\right)^{n}$ rather than $\left(a m_{\mathrm{b}}\right)^{n}$. Analogously, local composite fields are introduced in the effective lattice theory. For instance, the NLO HQET expansion of the zero-momentum projected time component of the heavy-light axial vector current can be written as

$$
\begin{aligned}
A_{0, \mathrm{R}}^{\mathrm{HQET}}\left(x_{0}\right) & =Z_{\mathrm{A}}^{\mathrm{HQET}} a^{3} \sum_{\mathbf{x}}\left[A_{0}^{\mathrm{stat}}(x)+c_{\mathrm{A}}^{(1)} A_{0}^{(1)}(x)\right] \\
A_{0}^{\mathrm{stat}}(x) & =\bar{\psi}_{1}(x) \gamma_{0} \gamma_{5} \psi_{\mathrm{h}}(x) \\
A_{0}^{(1)}(x) & =\bar{\psi}_{1}(x) \gamma_{5} \gamma_{i} \frac{1}{2}\left(\nabla_{i}^{\mathrm{S}}-\overleftarrow{\nabla}_{i}^{\mathrm{S}}\right) \psi_{\mathrm{h}}(x)
\end{aligned}
$$

$\nabla_{i}^{\mathrm{S}}$ being the spatial covariant derivative. The relation $f_{\mathrm{PS}} \sqrt{m_{\mathrm{PS}}}=\left\langle 0\left|A_{0, \mathrm{R}}(0)\right| \mathrm{PS}(\mathbf{p}=0)\right\rangle$ to the pseudoscalar decay constant will be used to calculate $f_{\mathrm{B}_{(\mathrm{s})}}$ below.

HQET treats the $\mathrm{O}\left(1 / m_{\mathrm{b}}\right)$ interactions terms in (1) as local space-time insertions in static correlations functions. For correlators of some multi-local fields $O$ and up to $1 / m_{\mathrm{b}}$-corrections to the operator itself (irrelevant when spectral quantities are considered), this means

$$
\begin{aligned}
\langle O\rangle=\langle O\rangle_{\text {stat }}+a^{4} \sum_{x}\{ & \omega_{\text {kin }}\left\langle O O_{\text {kin }}(x)\right\rangle_{\text {stat }} \\
& \left.+\omega_{\text {spin }}\left\langle O O_{\text {spin }}(x)\right\rangle_{\text {stat }}\right\},
\end{aligned}
$$

where $\langle O\rangle_{\text {stat }}$ is the expectation value in the static theory.

Still, for lattice HQET applications to lead to precise and controlled results, two issues had to be solved.
1.) The exponential growth of the noise-to-signal ratio in static-light correlation functions with Euclidean time, caused by the linear divergence in the binding energy $E^{\text {stat }}$ of the static-light system, which is particularly severe for the Eichten-Hill action [9]. This is overcome by so-called "HYP-smeared" [10] discretizations of the static quark action, improving the statistical precision of the correlators substantially [11, 12].

2.) Operator mixing in the effective theory induces UV power divergences in the lattice spacing that must be subtracted non-perturbatively: The formal definition of lattice HQET and its composite fields in (1) and (2) involves the (a priori free) effective couplings

$$
\omega \equiv\left\{m_{\mathrm{bare}}, \ln Z_{\mathrm{A}}^{\mathrm{HQET}}, c_{\mathrm{A}}^{(1)}, \omega_{\mathrm{kin}}, \omega_{\mathrm{spin}}\right\} .
$$

Here, the energy shift $m_{\text {bare }}$ is an additive mass renormalization. It absorbs the $1 / a$-divergence of the static energy, $E^{\text {stat }}$, and a $1 / a^{2}$-divergence at $\mathrm{O}\left(1 / m_{\mathrm{b}}\right)$. Hence, a phenomenologically relevant predictive power of lattice HQET is only guaranteed, once these HQET parameters $\omega=\left\{\omega_{i}\right\}$ have been fixed non-perturbatively such that no uncancelled power divergences in $1 / a$, which would remain in perturbation theory [13], can preclude to take the continuum limit.

A solution to 2.) was developed in [14] and relies upon a non-perturbative matching of HQET and QCD in finite volume. The implementation of this strategy by our collaboration has led to NLO HQET computations of the b-quark mass, B-meson spectroscopy and decay constants in the quenched approximation $\left(N_{\mathrm{f}}=0\right)$ [15[18], as well as in the more realistic two-flavour theory [19.-22], of which we give an overview in the following.

\subsection{General strategy}

The computational strategy of our approach [14, 16, 22], in which matching and renormalization are performed simultaneously and non-perturbatively ${ }^{2}$ is sketched in Fig. 2. The matching part is performed in a small volume of $L_{1} \approx 0.4 \mathrm{fm}$, where owing to $a m_{\mathrm{b}} \ll 1$ numerical simulations with a relativistic b-quark are feasible. The bare HQET parameters $\omega_{i}$ of the Lagrangian and the time component of the heavy-light axial current are fixed by imposing matching conditions $\Phi^{\mathrm{HQET}}(z, a) \stackrel{!}{=} \Phi^{\mathrm{QCD}}(z, 0)=\lim _{a \rightarrow 0} \Phi^{\mathrm{QCD}}(z, a)$. The

\footnotetext{
${ }^{2}$ As soon as $1 / m_{\mathrm{b}}$-corrections are included, matching must be done non-perturbatively in order not to spoil the asymptotic convergence of the series. Otherwise, the perturbative truncation error from the matching coefficient of the static term becomes much larger than the power corrections $\sim \Lambda_{\mathrm{QCD}} / m_{\mathrm{b}}$ of HQET, as $m_{\mathrm{b}} \rightarrow \infty$.
} 


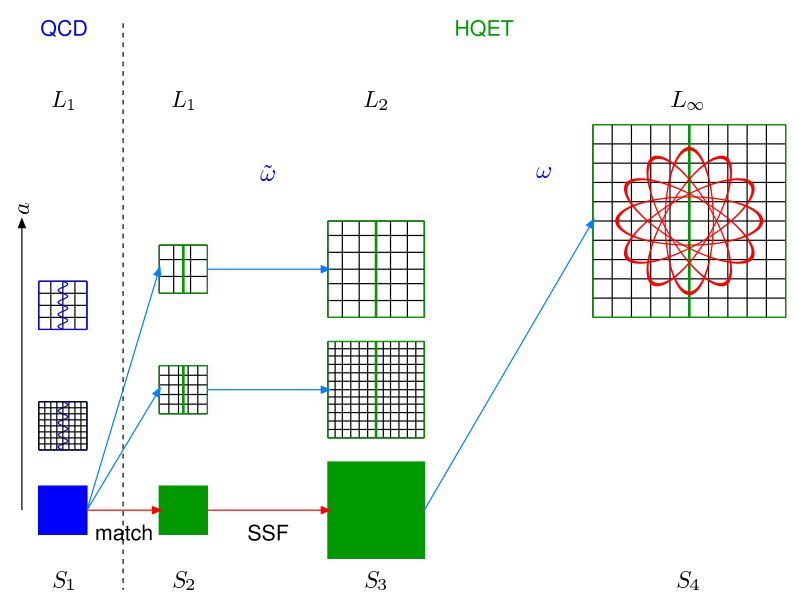

Figure 2: Idea of lattice HQET computations for B-physics phenomenology via a non-perturbative determination of HQET parameters from small-volume QCD simulations. The step scaling method makes contact to physically large volumes $L_{\infty}$. The whole construction is such that the continuum limit can be taken for all pieces.

quark mass dependence of (non-perturbatively renormalized) QCD is inherited by the HQET parameters $\omega_{i}$. It enters through the dimensionless variable $z \equiv L_{1} M$, where $M$ is the renormalization group invariant (RGI) mass [20]. Then a recursive finite-size scaling step $L_{1} \rightarrow L_{2}=2 L_{1}$ is used to reach larger volumes and lattice spacings $a$, by which connection with phenomenology in $L_{\infty} \gtrsim \max \left(2 \mathrm{fm}, 4 / m_{\pi}\right)$ can be made. As a result of [22], the HQET parameters (4), $\omega=\omega(z, a)$, absorbing the logarithmic and power divergences of HQET, are now non-perturbatively known for renormalized QCD quark masses from the charm to beyond the beauty region (parameterised by $z \in\{4,6,7,9,11,13,15,18,21\}$ ) and for $a$ 's corresponding to the bare gauge couplings of the available two-flavour configuration ensembles in large volume used to compute B-physics observables.

\subsection{Large-volume computations and techniques}

Our large-volume gauge configuration ensembles are characterised by the plaquette gauge action and a sea of $N_{\mathrm{f}}=2$ mass-degenerate non-perturbatively $\mathrm{O}(a)$ improved dynamical Wilson quarks. To be able to extrapolate to the physical pion mass, several pseudoscalar (sea) masses in the range $\left(190 \lesssim m_{\mathrm{PS}} \lesssim 440\right) \mathrm{MeV}$ with $L m_{\mathrm{PS}} \gtrsim 4$ are considered such that finite-volume effects are expected to be negligible. Moreover, the configurations cover $3 \beta$-values $\{5.2,5.3,5.5\}$ with lattice spacings $a \in\{0.08 \mathrm{fm}, 0.07 \mathrm{fm}, 0.05 \mathrm{fm}\}$ [23] to control the extrapolation to the continuum limit.
For the numerical simulations to generate these twoflavour QCD configurations, we employ M. Lüscher's implementation of the Hybrid Monte Carlo (HMC) algorithm with domain decomposition [24] and an adaption [25], which combines the deflated SAP solver [2628] with mass preconditioning [29], chronological inversion [30] and multiple time scale integration [31, 32]. Large trajectory length [33, 34] and long runs are used to ensure that our ensembles are not biased by the critical slowing down of the QCD simulations. For a careful and conservative error estimation, a binned jackknife procedure is applied (which is being cross-checked by the method advocated in [34]). All large-volume configuration ensembles have been produced and are shared within the Coordinated Lattice Simulations (CLS) effort by several lattice QCD teams in Europe [35].

Our determination of the B-meson spectrum and decay constants in two-flavour QCD is based on their HQET expansions in terms of the known HQET parameters and associated HQET energies and matrix elements at the static and $1 / m_{\mathrm{b}}-$ order. The latter are extracted from static-light correlation functions evaluated on the available large-volume CLS ensembles by solving the Generalised Eigenvalue Problem (GEVP) discussed and applied in [36, 37, 17, 18], allowing for a better control of excited state contaminations of the correlators. More specifically, the GEVP analysis amounts to compute a $(N \times N)$-matrix of correlators with the desired static and $\mathrm{O}\left(1 / m_{\mathrm{b}}\right)$ insertions, where each entry of the matrix corresponds to a different Gaussian smearing level [38] of the light quark field in the B-meson interpolating quark bilinear. In these computations, variance reduction in the light quark sector is achieved by stochastic all-to-all propagators (with 8 noise sources and full time-dilution) [39, 17], while for the static quark propagators, we use two variants of the HYPsmeared static actions, HYP1/2 [11, 12], already mentioned in Sect.2 Solving the GEVP numerically gives rise to new estimators for effective energies and hadronto-vacuum matrix elements, which converge faster with Euclidean time separation than standard ratios, since a larger gap governs the excited state corrections. I.e., corrections to ground state energies and matrix elements fall off in $t$ and $t_{0}$ as $\propto \exp \left\{-\left(E_{N+1}-E_{1}\right) t\right\}$ and $\propto \exp \left\{-\left(E_{N+1}-E_{1}\right) t_{0}\right\} \times \exp \left\{-\left(E_{2}-E_{1}\right)\left(t-t_{0}\right)\right\}$, respectively, where $t_{0}<t<2 t_{0}$ and $N$ labels the $N^{\text {th }}$ excited state (and $N=3$ in practice). Our final estimates are then obtained as plateau averages over ranges conservatively chosen by varying $t_{\min }$ for fixed $t_{\max }$ such that $\mathrm{O}\left(\mathrm{e}^{-\left(E_{N+1}-E_{1}\right) t}\right) \sim \sigma_{\text {sys }} \lesssim \frac{1}{3} \sigma_{\text {stat }}$, thereby minimising our systematic errors. For more details we refer to [37, 17, 18, 40, 41]. 


\section{Results}

In this section we summarise the status of results of our $N_{\mathrm{f}}=2$ B-physics project, as it was reported at summer conferences in 2012, see also [40, 42]. A final account of our work will be given later, once the full statistics of all CLS ensembles has been analysed [41].

Our HQET energies and matrix elements extracted from static-light correlators split into two sets, one for the B-meson sector with the valence quark masses set equal to the CLS sea quark mass values, and another one for the $\mathrm{B}_{\mathrm{s}}$-meson sector with the valence quark tuned to the physical strange quark [23], corresponding to a partially quenched setup.

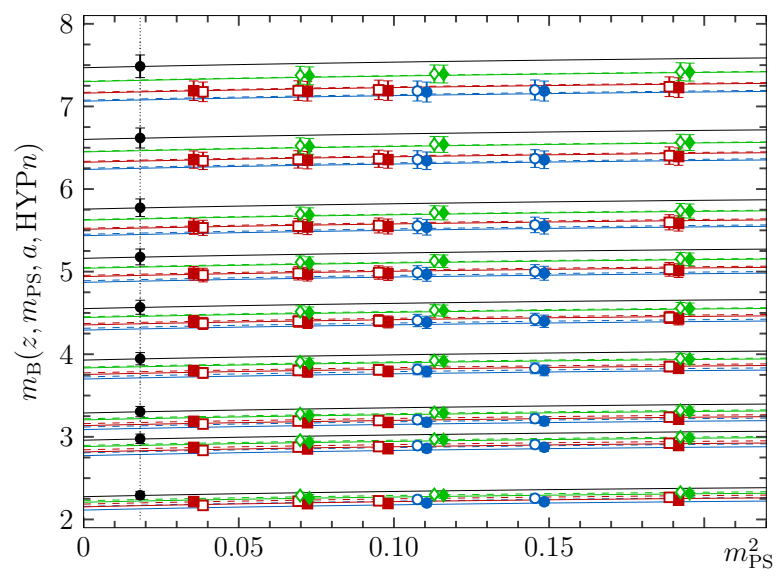

Figure 3: Joint chiral and continuum extrapolation of the heavy-light pseudoscalar meson mass (5) in NLO HQET to the ansatz (7) for fixed $z$. Recall that the $z$-dependence originates from the initial matching step to finite-volume QCD determining the HQET parameters. (Blue, red and green points refer to $\beta=5.2,5.3$ and 5.5, respectively, while filled/open symbols belong to the HYP1/2 static actions.)

\subsection{The b-quark's mass}

To begin with, we apply the non-perturbative results on the HQET parameters from the matching step together with the HQET energies from the large-volume GEVP analysis of the CLS ensembles to calculate the b-quark mass. To this end one writes down the NLO HQET expansion (i.e., to first order in $1 / m_{\mathrm{b}}$ ) of the heavy-light pseudoscalar meson mass as

$$
m_{\mathrm{B}}=m_{\mathrm{bare}}+E^{\mathrm{stat}}+\omega_{\mathrm{kin}} E^{\mathrm{kin}}+\omega_{\mathrm{spin}} E^{\mathrm{spin}} .
$$

Beside the dependence on the light pseudoscalar (sea) mass $m_{\mathrm{PS}}$ and the lattice spacing $a$ of the CLS configurations, we also have to account for the apparent heavy quark mass $(z)$ dependence of the HQET parameters. In fact, this $z$-dependence can now be exploited to fix the HQET parameters $\omega_{i}=\omega_{i}(z, a)$ for once by imposing the condition

$$
\left.m_{\mathrm{B}}\left(z, m_{\pi}, a\right)\right|_{z=z_{\mathrm{b}}} \equiv m_{\mathrm{B}}^{\exp }=5279.5 \mathrm{MeV}
$$

in the continuum, which defines the physical value of the b-quark mass at NLO of HQET.

For given $z$, the 1.h.s. is obtained by evaluating (5) for each value of the lattice spacing and sea quark mass of the CLS ensembles, followed by a global fit of $m_{\mathrm{B}}$ (simultaneously for two variants of the aforementioned HYP-smeared actions, $\operatorname{HYP} n, n=1,2$ ) to the ansatz for a combined chiral $\left(m_{\mathrm{PS}} \rightarrow m_{\pi} \equiv m_{\pi}^{\text {exp }}=135 \mathrm{MeV}\right.$ [1]) and continuum $(a \rightarrow 0)$ extrapolation,

$$
\begin{aligned}
& m_{\mathrm{B}}\left(z, m_{\mathrm{PS}}, a, \mathrm{HYP} n\right)= \\
& B(z)+C m_{\mathrm{PS}}^{2}-\frac{3 \hat{g}^{2}}{16 \pi f_{\pi}^{2}} m_{\mathrm{PS}}^{3}+D_{\mathrm{HYP} n} a^{2},
\end{aligned}
$$

with $f_{\pi} \equiv f_{\pi}^{\text {exp }}=130.4 \mathrm{MeV}$ [1] and $\hat{g}=0.51(2)$ [43] the $\mathrm{B}^{*} \mathrm{~B} \pi$-coupling in the static approximation for the b-quark. These extrapolations to the physical point are shown in Fig. 3

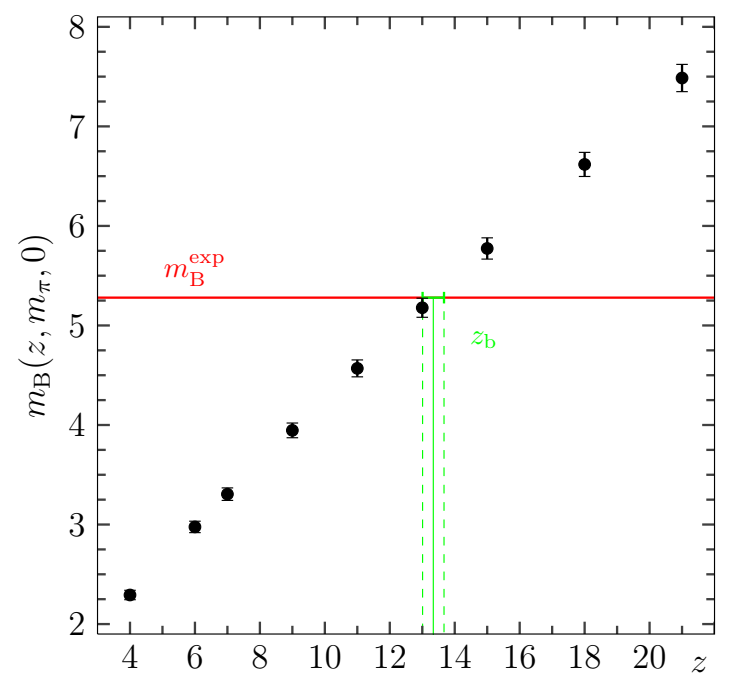

Figure 4: $z$-dependence of $m_{\mathrm{B}}$ in the continuum limit and graphical solution of [6, which determines the physical b-quark mass $z_{\mathrm{b}} .(z=$ $L_{1} M$ denotes the dimensionless RGI heavy quark mass.)

The solution of (6) for $a=0$ as the physical condition defining the b-quark mass yields the dimensionless RGI b-quark mass $z_{\mathrm{b}} \equiv L_{1} M_{\mathrm{b}}$ and is illustrated in Fig. 4. Converting $L_{1}$ to physical units, achieved via setting the lattice scale through $f_{\mathrm{K}}$ in [23], and translating with 4-/3-loop RG running of the coupling/mass to 
the conventional $\overline{\mathrm{MS}}$ scheme, we obtain as our result for the b-quark's mass in HQET at $\mathrm{O}\left(1 / m_{\mathrm{b}}\right)$ in the $N_{\mathrm{f}}=2$ theory presented at this year's summer conferences:

$$
\begin{aligned}
& z_{\mathrm{b}}=13.34(33)(13)_{z} \\
\Leftrightarrow \quad & \bar{m}_{\mathrm{b}} \overline{\mathrm{MS}}\left(\bar{m}_{\mathrm{b}}\right)=4.22(10)(4)_{z} \mathrm{GeV} .
\end{aligned}
$$

The first error covers all statistical and systematic errors, including those from the GEVP analysis, the various extrapolations and the scale setting, while the second uncertainty of about $1 \%$ stems from the quark mass renormalization in QCD, entering the finite-volume matching step [20, 22], and has to be added in quadrature. Since we find the difference of the NLO HQET result (8) and the corresponding number in the static approximation (LO HQET) to be very small, we conclude that the truncation error of $\mathrm{O}\left(\Lambda_{\mathrm{QCD}}^{3} / m_{\mathrm{b}}^{2}\right)$ to 8 in the HQET expansion is negligible compared to our overall error.

Our result (8) also compares very well with other recent determinations and the value quoted by the Particle Data Group, see the (incomplete) compilation in Tab. 1 Note that some determinations claim very small errors, although they are based on perturbation theory or lattice data with heavy quark masses in lattice units close to 1 .

\begin{tabular}{lll}
\hline $\bar{m}_{\mathrm{b}}^{\overline{\mathrm{MS}}}\left(\bar{m}_{\mathrm{b}}\right) / \mathrm{GeV}$ & remarks, method & ref. \\
\hline $4.347(48)$ & lattice, $N_{\mathrm{f}}=0, \mathrm{NLO} \mathrm{HQET}$ & \\
$4.22(11)$ & lattice, $N_{\mathrm{f}}=2, \mathrm{NLO} \mathrm{HQET}$ & eq. \\
$4.29(14)$ & lattice, $N_{\mathrm{f}}=2$, extrapolation & \\
$4.164(23)$ & lattice, $N_{\mathrm{f}}=3$, extrapolation \\
$4.163(16)$ & perturbation theory \& data \\
$4.236(69)$ & perturbation theory \& QCD inputs \\
$4.18(3)$ & PDG average 2012
\end{tabular}

Table 1: Compilation of some recent determinations of $m_{\mathrm{b}}$. As for the lattice results [44] and 45], the former uses extrapolations of relativistic data around the charm to known static limits, while the latter employs moments of current-current correlators with Highly Improved Staggered Quarks (HISQ) [48] extrapolated to the b-scale. [46] and 477 rely on QCD sum rules. For more details, see the cited references.

After the determination of the value of the physical bquark mass (and thus $z_{\mathrm{b}}$ ) from $m_{\mathrm{B}}$, we can fix the HQET parameters to $\omega_{i}(a)=\omega_{i}\left(z_{\mathrm{b}}, a\right)$ and use those in any successive HQET computation of B-physics obervables.

\subsection{B-meson decay constants}

To determine the B-meson decay constants $f_{\mathrm{B}}$ and $f_{\mathrm{B}_{\mathrm{s}}}$, we now combine the HQET parameters with the ma- trix elements resulting from the GEVP analysis. Distinguishing the heavy-light $\mathrm{B}_{\mathrm{r}}$-meson decay constants, where the light flavour can be either a valence (= sea) quark flavour $\mathrm{r}=\{\mathrm{u}, \mathrm{d}\} \equiv 1$ or a valence strange one, $\mathrm{r}=\mathrm{s}$, their NLO HQET expansions in terms of the HQET parameters $\omega_{i}$ read

$$
\begin{aligned}
f_{\mathrm{B}_{\mathrm{r}}} \sqrt{\frac{m_{\mathrm{Br}}}{2}}= & Z_{\mathrm{A}}^{\mathrm{HQET}}\left(1+b_{\mathrm{A}}^{\text {stat }} a m_{\mathrm{q}, \mathrm{r}}\right) p_{\mathrm{r}}^{\text {stat }} \\
& \times\left(1+\omega_{\mathrm{kin}} p_{\mathrm{r}}^{\mathrm{kin}}+\omega_{\mathrm{spin}} p_{\mathrm{r}}^{\text {spin }}+c_{\mathrm{A}}^{(1)} p_{\mathrm{r}}^{\mathrm{A}^{(1)}}\right) .
\end{aligned}
$$

As explained before, the $\omega_{i}$ are understood to be taken at the physical b-quark mass, $z_{\mathrm{b}}$, and the $p^{\mathrm{X}}, \mathrm{X} \in$ \{stat, kin, spin, $\mathrm{A}^{(1)}$, denote the previously extracted GEVP plateau values of the associated effective matrix elements of Sect.2.2. The improvement coefficient $b_{\mathrm{A}}^{\text {stat }}$ is known to $1-$ loop perturbation theory ${ }^{3}$ from [49].

Due to non-perturbative $\mathrm{O}(a)$ improvement employed in our computations, $f_{\mathrm{B}_{\mathrm{r}}}$ (with $\mathrm{r}=1$, s and $f_{\mathrm{B}_{1}} \equiv f_{\mathrm{B}}$ ), approaches the continuum limit quadratically in the lattice spacing. In order to estimate a systematic error in our combined chiral and continuum extrapolation, we choose fits, where the sea quark dependence is modelled according to the prediction of Heavy Meson Chiral Perturbation Theory $(\mathrm{HM} \chi \mathrm{PT})[50,51]$, as well as only linear in $m_{\mathrm{PS}}^{2}$,

$$
\begin{aligned}
& f_{\mathrm{B}}\left(m_{\mathrm{PS}}, a, \mathrm{HYP} n\right)= \\
& b^{\prime}\left[1-\frac{3}{4} \frac{1+3 \hat{g}^{2}}{\left(4 \pi f_{\pi}\right)^{2}} m_{\mathrm{PS}}^{2} \ln \left(m_{\mathrm{PS}}^{2}\right)\right]+c^{\prime} m_{\mathrm{PS}}^{2}+d_{\mathrm{HYP} n}^{\prime} a^{2}, \\
& f_{\mathrm{B}_{\mathrm{r}}}\left(m_{\mathrm{PS}}, a, \mathrm{HYP} n\right)=b_{\mathrm{r}}+c_{\mathrm{r}} m_{\mathrm{PS}}^{2}+d_{\mathrm{r}, \mathrm{HYP} n} a^{2} ;
\end{aligned}
$$

here, $f_{\pi}=f_{\pi}^{\exp }$ and $\hat{g}=0.51(2)$ are the same as above. These joint extrapolations are depicted as the black solid curves in Figs. 5 and 6 In particular for $f_{\mathrm{B}_{\mathrm{s}}}$, not all CLS ensembles were analysed yet.

From the figures one can infer that whether we do or do not include the chiral logarithm of $\mathrm{HM} \chi \mathrm{PT}$ in the extrapolation 10 of $f_{\mathrm{B}}$ induces a very small change at the physical point only. We thus take the $\mathrm{HM} \chi \mathrm{PT}$ extrapolation as the central value and the difference to the linear fit to account for a part of the systematic error of our final result. For the $\mathrm{B}-$ and $\mathrm{B}_{\mathrm{s}}$-meson decay constants from HQET at $\mathrm{O}\left(1 / m_{\mathrm{b}}\right)$ in two-flavour QCD we preliminarily give

$$
\begin{aligned}
f_{\mathrm{B}} & =193(9)(4)_{\chi} \mathrm{MeV} \\
f_{\mathrm{B}_{\mathrm{s}}} & =219(12) \mathrm{MeV}
\end{aligned}
$$

\footnotetext{
${ }^{3}$ For the subtracted bare quark masses appearing here, the additive improvement term $b_{\mathrm{A}}^{\text {stat }} a m_{\mathrm{q}, \mathrm{r}}$ is numerically very small in practice.
} 


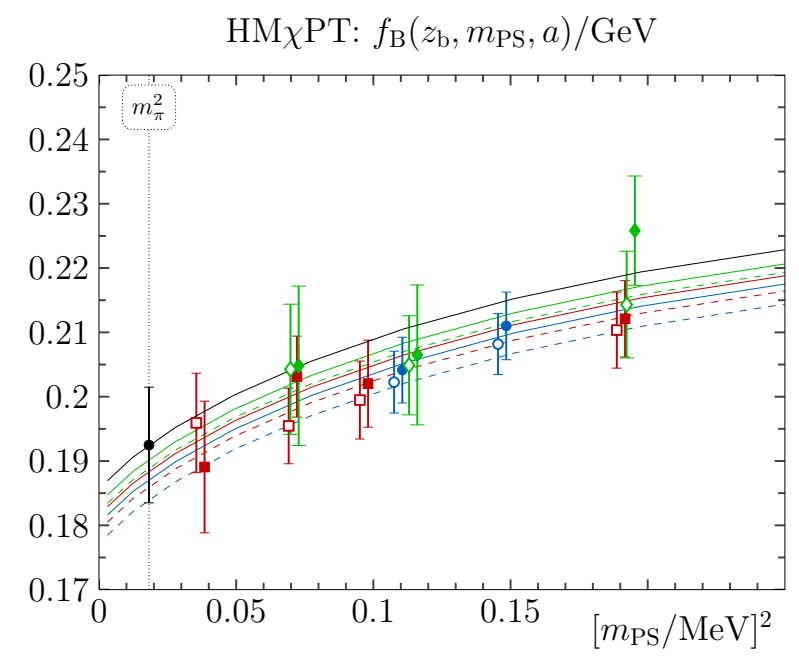

Figure 5: Joint chiral and continuum extrapolation to the physical point of the B-meson decay constant (9) in NLO HQET to the $H M \chi P T-m o t i v a t e d$ ansatz [10]. The colour coding is the same as in Fig. 3 (I.e., blue, red and green points refer to $\beta=5.2,5.3$ and 5.5, while filled/open symbols belong to the HYP1/2 static actions.)

where the quoted errors again cover all sources of statistical and systematic uncertainties.

Our results $(12)$ and $(13)$ are in line with computations of other groups, see, e.g., summaries in [52,47].

\section{Outlook}

The non-perturbative treatment of NLO HQET with controlled chiral and continuum extrapolations leads to results for B-physics phenomenology with a few-\% accuracy. They can contribute to resolving current tensions in precision CKM analyses of the B-meson sector. As our computations are the only ones, which have no perturbative uncertainties, including the renormalization of the axial current, this introduces a new quality, albeit for $N_{\mathrm{f}}=2$.

We are also investigating further spectral quantities within our approach, for instance, the B-meson spin splittings. Owing to the heavy quark spin-symmetry, the mass difference between the vector $\mathrm{B}^{*}$ - and the pseudoscalar B-meson is dominated by a pure $\mathrm{O}\left(1 / m_{\mathrm{b}}\right)$ effect from the contribution of $O_{\text {spin }}$ to the effective HQET Lagrangian (1) and, hence, is of particular interest.

By extending our finite-volume matching strategy to all components of the axial and vector currents, we aim at a NLO HQET calculation of $\mathrm{B} \rightarrow \pi$ semi-leptonic decay form factors as possible application. A status report in the LO (static) approximation has been given in [53].

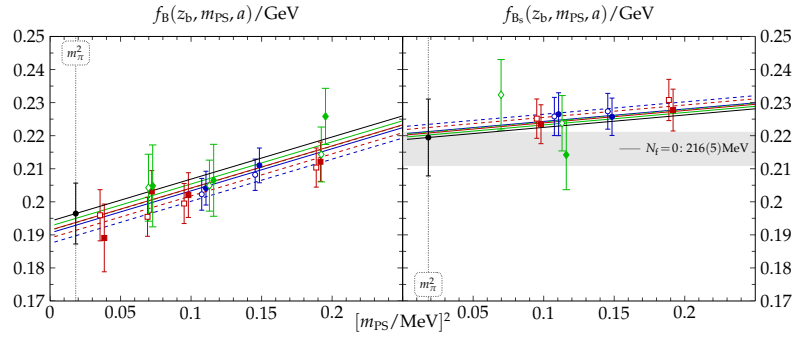

Figure 6: Joint chiral and continuum extrapolation to the physical point of $f_{\mathrm{B}}$ (left) and $f_{\mathrm{B}_{\mathrm{S}}}$ (right) in NLO HQET, where only a linear dependence on the squared light pseudoscalar (sea) mass $m_{\mathrm{PS}}^{2}$ is assumed, cf. [11). In case of $f_{\mathrm{B}_{\mathrm{s}}}$, the NLO HQET result $f_{\mathrm{B}_{\mathrm{s}}}=$ $216(5) \mathrm{MeV}$ obtained in the quenched approximation $\left(N_{\mathrm{f}}=0\right)$ [18] (where the scale was set through $r_{0}=0.5 \mathrm{fm}$ ) is included for comparison. The colour coding is the same as in Fig. 5

\section{Acknowledgments}

This work is supported by the DFG in the SFB/TR 9, "Computational Particle Physics", and was formerly so through EU Contract No. MRTN-CT-2006-035482, "FLAVIAnet". We thank for further funding by the grants STFC ST/G000522/1 and EU "STRONGnet" 238353 (N. G.), and DFG HE 4517/2-1 (P. F. and J. H.). We are indebted to our colleagues in CLS for the joint production and use of $N_{\mathrm{f}}=2$ configurations and also acknowledge the computer resources provided by the NIC at FZ Jülich, HLRN in Berlin and DESY, Zeuthen, where most of our simulations have been performed.

\section{References}

[1] Particle Data Group, J. Beringer et al., Phys. Rev. D86 (2012) 010001, http://pdg.lbl.gov.

[2] BELLE, Y. Yook, talk at ICHEP 2012.

[3] BELLE, I. Adachi et al., 1208.4678.

[4] E. Lunghi and A. Soni, Phys. Lett. B697 (2011) 323, 1010.6069.

[5] J. Laiho, E. Lunghi and R. Van de Water, PoS LATTICE2011 (2011) 018, 1204.0791.

[6] CKMfitter, A. Lenz et al., Phys. Rev. D86 (2012) 033008, 1203.0238 .

[7] E. Eichten and B. Hill, Phys. Lett. B234 (1990) 511.

[8] E. Eichten and B. Hill, Phys. Lett. B243 (1990) 427.

[9] E. Eichten and B. Hill, Phys. Lett. B240 (1990) 193

[10] A. Hasenfratz and F. Knechtli, Phys. Rev. D64 (2001) 034504, hep-lat/0103029.

[11] ALPHA, M. Della Morte et al., Phys. Lett. B581 (2004) 93, hep-lat/0307021, Erratum: ibid. B612 (2005) 313.

[12] ALPHA, M. Della Morte, A. Shindler and R. Sommer, J. High Energy Phys. 08 (2005) 051, hep-lat/0506008.

[13] L. Maiani, G. Martinelli and C.T. Sachrajda, Nucl. Phys. B368 (1992) 281.

[14] ALPHA, J. Heitger and R. Sommer, J. High Energy Phys. 02 (2004) 022, hep-lat/0310035.

[15] ALPHA, M. Della Morte et al., J. High Energy Phys. 01 (2007) 007, hep-ph/0609294. 
[16] ALPHA, B. Blossier et al., J. High Energy Phys. 06 (2010) 002, 1001.4783.

[17] ALPHA, B. Blossier et al., J. High Energy Phys. 05 (2010) 074, 1004.2661.

[18] ALPHA, B. Blossier et al., J. High Energy Phys. 12 (2010) 039, 1006.5816.

[19] ALPHA, M. Della Morte et al., PoS LATTICE2008 (2008) 226, 0810.3166 .

[20] ALPHA, P. Fritzsch, J. Heitger and N. Tantalo, J. High Energy Phys. 08 (2010) 074, 1004.3978.

[21] ALPHA, B. Blossier et al., PoS LATTICE2011 (2011) 280, 1112.6175 .

[22] ALPHA, B. Blossier et al., J. High Energy Phys. 09 (2012) 132, 1203.6516

[23] ALPHA, P. Fritzsch et al., Nucl. Phys. B865 (2012) 397, 1205.5380 .

[24] M. Lüscher, Comput. Phys. Commun. 165 (2005) 199, heplat/0409106.

[25] ALPHA, M. Marinkovic and S. Schaefer, PoS LATTICE2010 (2010) 031, 1011.0911

[26] M. Lüscher, Comput. Phys. Commun. 156 (2004) 209, heplat/0310048.

[27] M. Lüscher, J. High Energy Phys. 07 (2007) 081, 0706.2298

[28] M. Lüscher, J. High Energy Phys. 12 (2007) 011, 0710.5417.

[29] M. Hasenbusch, Phys. Lett. B519 (2001) 177, hep-lat/0107019.

[30] R.C. Brower et al., Nucl. Phys. B484 (1997) 353, heplat/9509012.

[31] J.C. Sexton and D.H. Weingarten, Nucl. Phys. B380 (1992) 665.

[32] C. Urbach et al., Comput. Phys. Commun. 174 (2006) 87, heplat/0506011.

[33] ALPHA, H.B. Meyer et al., Comput. Phys. Commun. 176 (2007) 91, hep-lat/0606004.

[34] ALPHA, S. Schaefer, R. Sommer and F. Virotta, Nucl. Phys. B845 (2011) 93, 1009.5228.

[35] https://twiki.cern.ch/twiki/bin/view/CLS/WebHome.

[36] M. Lüscher and U. Wolff, Nucl. Phys. B339 (1990) 222.

[37] ALPHA, B. Blossier et al., J. High Energy Phys. 04 (2009) 094, 0902.1265.

[38] S. Güsken et al., Phys. Lett. B227 (1989) 266.

[39] J. Foley et al., Comput. Phys. Commun. 172 (2005) 145, heplat/0505023.

[40] ALPHA, F. Bernardoni et al., to appear in the proceedings of LATTICE 2012 (PoS).

[41] ALPHA, in preparation.

[42] ALPHA, F. Bahr et al., to appear in the proceedings of ICHEP $2012(\mathrm{PoS})$

[43] ALPHA, J. Bulava, M. Donnellan and R. Sommer, PoS LATTICE2010 (2010) 303, 1011.4393.

[44] ETMC, P. Dimopoulos et al., J. High Energy Phys. 01 (2012) 046, 1107.1441.

[45] HPQCD, C. McNeile et al., Phys. Rev. D82 (2010) 034512, 1004.4285.

[46] K.G. Chetyrkin et al., Phys. Rev. D80 (2009) 074010, 0907.2110.

[47] S. Narison, 1209.2023, 1209.2925 and these proceedings.

[48] HPQCD, E. Follana et al., Phys. Rev. D75 (2007) 054502, heplat/0610092.

[49] A. Grimbach et al., J. High Energy Phys. 08 (2008) 039, 0802.0862.

[50] J.L. Goity, Phys. Rev. D46 (1992) 3929, hep-ph/9206230.

[51] S.R. Sharpe and Y. Zhang, Phys. Rev. D53 (1996) 5125, heplat/9510037.

[52] C. Davies, PoS LATTICE2011 (2011) 019, 1203.3862.

[53] ALPHA, F. Bahr et al., 1210.3478, to appear in the proceedings of LATTICE 2012 (PoS) 